\title{
LIPIDOS TOTALES EN LA MUSCULATURA DE PINGUIPES CHILENSIS VALENCIENNES 1833 (PERCIFORMES, PINGUIPEDIDAE)
}

\section{TOTAL LIPIDS IN MUSCLE OF PINGUIPES CHILENSIS VALENCIENNES 1833 (PERCIFORMES, PINGUIPEDIDAE)}

\author{
Paola González ${ }^{1}$ Ciro Oyarzún² \& José Becerra ${ }^{3}$ \\ ${ }^{1}$ Programa Doctorado en Ciencias Biológicas, Area Zoología, Facultad de Ciencias Naturales y Oceanográficas \\ paogonza@udec.cl \\ ${ }^{2}$ Depto. de Oceanografía. Universidad de Concepción \\ ${ }^{3}$ Depto. de Botánica. Universidad de Concepción, Casilla 160-C, Concepción. Chile. Email:
}

\section{RESUMEN}

Pinguipes chilensis presenta un comportamiento alimentario y reproductivo que comprende todo el año, en donde índices de condición como Indice Hepatosomático y Factor de Condición no se relacionan con la madurez de las gónadas, indicando una posible independencia de la energía almacenada como lípidos en la musculatura y en el hígado con el proceso reproductivo. El presente trabajo determina el contenido de lípidos totales (\%) en la musculatura de P. chilensis, y su variación entre zonas del cuerpo, así como diferencias entre sexos y ontogenéticas. En hembras (n=12) y machos (n=11) capturados en el litoral de Talcahuano, Chile, se determinó el contenido de lípidos totales mediante la Técnica Gravimétrica de extracción por Soxhlet, de tejido muscular desde tres zonas del cuerpo, anterior, medio (con y sin tegumento) y posterior. La musculatura de hembras y machos no muestra diferencias significativas en el contenido de lípidos totales por zona del cuerpo del pez analizado ( $\mathrm{p}=0,12$ y p=0,24, respectivamente). En muestras agrupadas, el contenido de lípidos de la zona posterior es menor $(\mathrm{p}=0,01)$ al de la zona media del pez. El porcentaje de lípidos totales no se relacionó con la talla $(p>0,20)$ y el peso $(p>0,20)$ de los individuos, reflejando una importancia menor de la musculatura como sitio de almacenaje de lípidos o una movilización continua de lípidos totales para procesos de maduración gonadal.

Palabras claves: Lípidos, Pinguipes chilensis, Chile.

\section{ABSTRACT}

Pinguipes chilensis presents an alimentary and reproductive pattern similar around the year. The Hepatosomatic Index and the Condition Factor are not correlated with gonad maturation as an expression of independence between store energy as lipids in muscles and liver associated with reproductive processes. In this work the percentage of total lipids in muscles of $P$. chilensis and body variation were determined. Also the differences between sex and ontogeny were evaluated. In fishes caught off Talcahuano, Chile (12 females and 11 males) the total lipids percentages were quantified using the Soxhlet method. Samples of muscles were taken from three parts of the body (anterior, center and posterior). The muscles present no statistical differences in lipid content between the body parts for males or females $(\mathrm{p}=0.24$ and 0.12 respectively). In pooled samples the lipid content in the posterior part is minor $(\mathrm{p}=0.01)$ to the central part. The total lipid contents are not related with length ( $p>0.2)$ or weight $(p>0.2)$ showing the muscles with less importance as lipids store or that the lipids are moved continuously for the gonad maturation.

Keywords: Lipids, Pinguipes chilensis, Chile. 


\section{INTRODUCCION}

La principal reserva energética en peces corresponde a lípidos, fundamentalmente triacilglicéridos, los cuales son consumidos en actividades de alta demanda energética, tales como la reproducción y migración, o en actividades de rutinas durante períodos de escasez de recursos (Schultz \& Conover 1999). La composición química de diferentes tejidos puede variar considerablemente, especialmente en el contenido de lípidos y agua (Haug 1990). Durante períodos de alta ingesta los lípidos pueden ser almacenados en el tejido somático y el hígado, así como en torno a las vísceras (Hoque et al. 1998, Collins \& Anderson 1995), por lo que los lípidos pueden ser usados como marcadores de condición, ya que pueden mostrar períodos de ciclos anuales asociados con cambios en las condiciones de los peces (Shulman \& Love 1999). Existirían cambios estacionales en la composición de lípidos, agua y proteínas en músculos, hígado y vísceras, asociados a procesos de crecimiento gonadal y otros procesos asociados al desove, ya que la acumulación de lípidos depende directamente de la disponibilidad de alimento, y es muy importante en la recuperación en períodos de postdesove (Eliassen \& Vahl 1982; Shulman \& Love 1999).

Pinguipes chilensis Valenciennes 1833 es una especie endémica del océano Pacífico Suroriental, que se distribuye desde las costas de Tumbes en Perú hasta el extremo austral de Chile. La información más reciente indica que presenta una alimentación compuesta por una gran diversidad trófica, y un período de desove que abarca todo el año, con máximos en las estaciones de verano y otoño (González \& Oyarzún 2003) y que los valores de Indice Hepatosomático (IHS) y el Factor de Condición (FCC) no se relacionan con el grado de madurez de las gónadas (IGS) (Gonzalez \& Oyarzún 2002). Lo anterior permitiría hipotetizar una posible independencia de la energía almacenada en la musculatura con la producción de ovocitos o una rápida movilización de la energía adquirida. En el presente trabajo se evaluó el porcentaje de lípidos totales en el tejido muscular de $P$. chilensis capturados en otoño y la posible relación con el sexo, la longitud y el peso de los individuos.

\section{MATERIALES Y METODOS}

Se analizó un total de 23 ejemplares (12 hembras y 11 machos) capturados en los meses de otoño de 2003 en el litoral de Talcahuano ( $36^{\circ} 41^{\prime} \mathrm{S}$; $\left.73^{\circ} 06^{\prime} \mathrm{W}\right)$. Para cada individuo se registró la longitud total (rango: 29,5-50,5 cm), peso eviscerado (rango: 304$1.654 \mathrm{~g}$ ) y sexo. En cada individuo se obtuvo una muestra aproximada de $10 \mathrm{~g}$ de tejido muscular blanco, de las zonas anterior, media (con y sin tegumento) y posterior del cuerpo. Cada muestra fue guardada a $-20^{\circ} \mathrm{C}$ hasta su posterior análisis. Mediante la técnica de extracción por Soxhlet, utilizando como solvente Hexano, se determinó gravimétricamente el contenido de lípidos totales presente en la musculatura, el cual se expresó como porcentaje en relación al peso del tejido muscular. Mediante Anova de una vía no paramétrica, KruskallWallis (K-W), se evaluó diferencias significativas en el contenido de lípidos entre sexos y zonas del cuerpo. Se complementó con un test a posteriori, (Prueba de Mann-Whitney, M-W) para evaluar diferencias entre zonas. Mediante regresión lineal se evaluó la relación del contenido de lípidos con la longitud total y el peso eviscerado de los ejemplares. El nivel de significancia empleado fue de 0.05 .

\section{RESULTADOS Y DISCUSION}

Al comparar el contenido de lípidos totales para las zonas del cuerpo analizadas, las hembras no muestran diferencias $(\mathrm{K}-\mathrm{W}, \mathrm{p}=0,122)$ al igual que en machos (K-W, $\mathrm{p}=0,247)$. Los valores de lípidos totales obtenidos para machos son iguales a los de las hembras (Tabla I), si bien se aprecia una tendencia de mayor contenido de lípidos en las hembras en las zonas del pez analizadas (Tabla I). Lo anterior concuerda con lo encontrado en Hippoglossus hippoglossus (Linnaeus 1758), donde las hembras alcanzan valores de contenido de lípidos muscular mayor que los machos, con una tendencia a la disminución de éste desde la zona anterior hacia la posterior (Haug et al. 1988). Como no existen diferencias por sexo en el contenido lipídico, se hizo un análisis del total de casos entre zonas, apareciendo diferencias entre ellas $(\mathrm{K}-\mathrm{W}, \mathrm{p}=0,013)$ (Figura 1), las diferencias están dada entre la zona posterior con la zona media con tegumento $(M-W, p=0,003)$ y media sin tegumento (M-W, $\mathrm{p}=0,027)$. 
Lípidos en Pinguipes chilensis. GonzáLez, P. ET AL

TABLA I. Contenido de lípidos totales (Porcentaje \pm desviación estándar) en hembras y machos de $P$. chilensis, en musculatura de tres zonas del cuerpo ( $\mathrm{ct}=$ con tegumento, $\mathrm{st}=$ sin tegumento).

TABLE I. Total lipids contents (Percentage \pm standard deviation) in females and males of $P$. chilensis for musculature from three zone of the body. $(\mathrm{ct}=$ with tegument, $\mathrm{st}=$ without tegument $)$.

\begin{tabular}{llll}
\hline Zonas del cuerpo & Hembras & Machos & $p$ \\
\hline Anterior $($ ct $)$ & $1,27(2,98)$ & $0,37(0,20)$ & 0,83 \\
Media $($ ct $)$ & $1,93(3,31)$ & $0,49(0,42)$ & 0,34 \\
Media $($ st $)$ & $2,08(4,59)$ & $1,43(2,34)$ & 0,77 \\
Posterior $($ ct $)$ & $0,22(0,19)$ & $0,47(0,69)$ & 0,55 \\
\hline
\end{tabular}

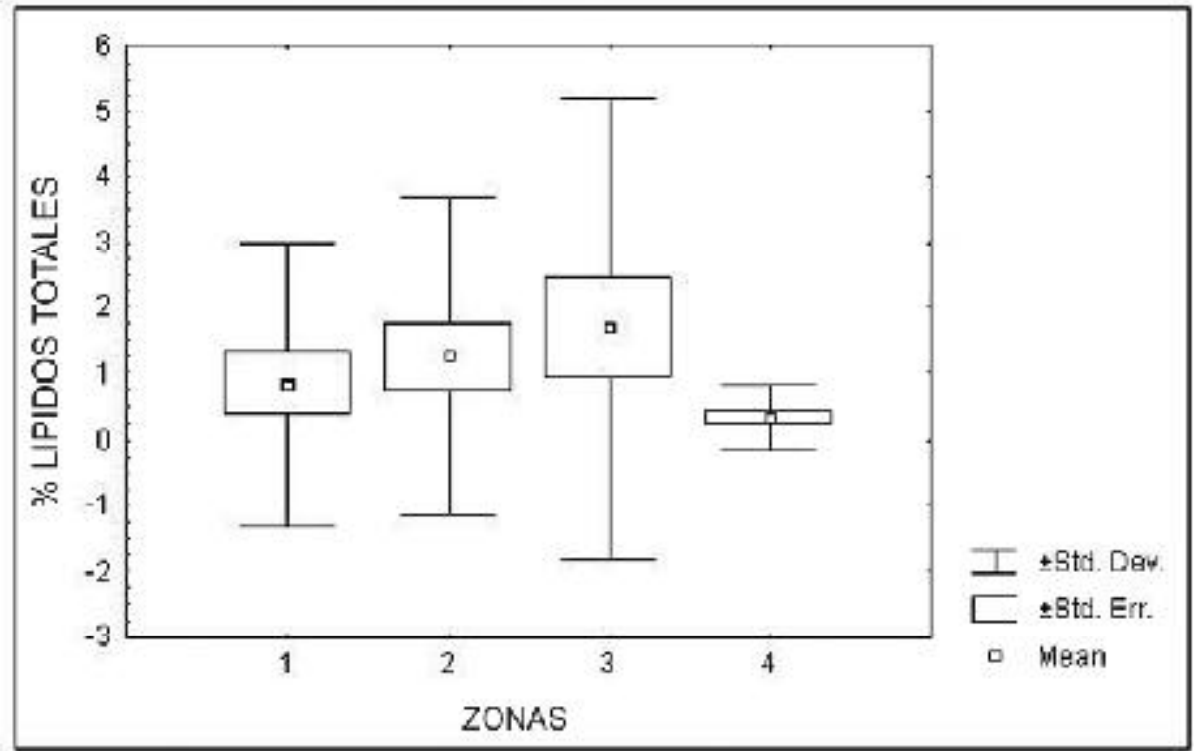

Figura 1. Contenido porcentual de lípidos totales de las zonas anterior (1), medio con (2) y sin (3) tegumento y posterior (4) en P. chilensis ( $1=$ anterior (ct), $2=$ medio (ct), $3=$ medio (st) y $4=$ posterior (ct)).

FIGURE 1. Total lipids contents as percentage of zones anterior (1), middle with (2) and without (3) tegument and posterior (4) of P. chilensis.

En $P$. chilensis los individuos más grandes presentan similar cantidad de energía almacenada en forma de lípidos que los individuos de menor tamaño. Al comparar los porcentajes de lípidos totales en función de la longitud total, o del peso eviscerado no existen diferencias significativas (Tabla II). Esto es contrario a lo señalado en cuanto a que la capacidad de almacenar energía es dependiente del tamaño del cuerpo como ocurre en Atherina boyeri Risso, 1810 y Menidia menidia (Linnaeus 1766), donde niveles tamaño específico de grasa generalmente se incrementan con el tamaño (Henderson et al. 1988, Schultz \& Conover 1997). P. chilensis es una especie con un comportamiento de alimentación constante durante todo el año, presentando una gran amplitud trófica (González \& Oyarzún 2003) y como la 
acumulación de lípidos depende directamente de la adquisición de alimento (Collins \& Anderson 1995; Shulman \& Love 1999), y la energía adquirida en ésta y almacenada en el músculo no muestra variaciones, puede estar indicando una importancia del hígado como principal sitio de almacenaje de reservas de energía en $P$. chilensis, como ha sido determinado en otras especies (Love 1980, Haug 1990) o una movilización continua de la energía almacenada en procesos de maduración, lo que no reflejaría diferencias del porcentaje de lípidos en la musculatura.

TABLA II. Valores de regresión entre el contenido de lípidos totales de la musculatura con (a) la longitud tota, y (b) el peso eviscerado en P. chilensis.

TABLE II. Regression values between total lipids contents in muscle with (a) total length and (b) eviscerated weight of $P$. chilensis

\begin{tabular}{lrlll}
\hline Zona & \multicolumn{1}{c}{$\mathrm{a}$} & $\mathrm{b}$ & $\mathrm{r}^{2}$ & $\mathrm{p}$ \\
\hline Longitud total $(\mathrm{cm})$ & & & & \\
Anterior & 2,414 & 0,088 & 0,023 & 0,51 \\
Media (c/t) & 0,135 & 0,038 & 0,004 & 0,84 \\
Media (s/t) & 10,144 & 0,221 & 0,078 & 0,21 \\
Posterior & 0,831 & 0,025 & 0,064 & 0,25 \\
Peso eviscerado (g) & & & & \\
Anterior & 0,039 & 0,001 & 0,015 & 0,57 \\
Media (c/t) & 0,963 & 0,0004 & 0,002 & 0,83 \\
Media (s/t) & 3,923 & 0,003 & 0,066 & 0,25 \\
Posterior & 0,123 & 0,0003 & 0,037 & 0,39 \\
\hline
\end{tabular}

\section{BIBLIOGRAFIA}

Collins, A. L. \& T. A. Anderson. 1995. The regulation of endogeneuos energy stores during starvation and refeeding in the somatic tissues of the golden perch. Journal of Fish Biology 47: 1004-1015.

ELIASSEN, J-E. \& O. VAHL. 1982. Seasonal variations in biochemical composition and energy content of liver, gonad and muscle of mature and immature cod, $\mathrm{Ga}$ dus morhua (L.) from Balsfjorden, northern Norway. Journal of Fish Biology 20: 707-716.

González, P. \& C. Oyarzún. 2002. Variabilidad de índices biológicos en Pinguipes chilensis Valenciennes 1833 (Perciformes, Pinguipedidae): ¿Están realmente correlacionados? Gayana 66 (2): 249253.

González, P. \& C. Orarzún. 2003. Diet of the Chilean sandperch, Pinguipes chilensis (Perciformes, Pinguipedidae) in southern Chile. Journal of Applied Ichthyology 19 (6): 371-375.

Haug, T., E. Ringo \& G WiBEChe. 1988. Total lipid and fatty acid composition of polar and neutral lipids in dif- ferent tissues of Atlantic halibut, Hippoglossus hippoglossus (L). Sarsia 73 (2): 163-168.

HaUG, T. 1990. Biology of the Atlantic Halibut Hippoglossus hippoglosus (L., 1758). Advances in Marine Biology 26: 1-70.

Henderson, P. A., R.H.A. Holmes \& R. N. Bamber. 1988. Size-selective overwintering mortality in the sand smelt, Atherina boyeri Risso, and its role in population regulation. Journal of Fish Biology 33(2): 2212303.

Hoque, M.T., F. M. Yusoff, A. T. Law \& M. A. Syed. 1998 Effect of hydrogen sulphide on liver somatic index and Fulton's condition factor in Mystus nemurus. Journal of Fish Biology 52: 23-30.

Love, R. M. 1980. The chemical Biology of Fishes. $2^{\text {nd }}$ edition. Academic Press, London.

Schultz, E. T. \& D. O. Conover. 1999. The allometry of energy reserve depletion: test of mechanism for sizedependent winter mortality. Oecologia 119: 474483.

Shulman, G. E. \& R. M. Love(editors). 1999. The biochemical Ecology of marine fishes. Advances in Marine Biology 36: 1 - 350. 